doi:10.13108/2015-7-3-9

\title{
ON A NEW APPROACH FOR STUDYING ASYMPTOTIC BEHAVIOR OF SOLUTIONS TO SINGULAR DIFFERENTIAL EQUATIONS
}

\author{
N.F. VALEEV, E.A. NAZIROVA, YA.T. SULTANAEV
}

\begin{abstract}
In the work we propose a new approach for studying the asymptotic behavior for large $x$ of the solutions to singular linear two-terms differential equations

$$
-\frac{d^{n}}{d x^{n}} y(x, \lambda)+\lambda q(x) y(x, \lambda)=0
$$

with a potential $q(x)$ growing non-regularly as $x \rightarrow \infty$. The idea of constructing the asymptotics for the solutions of singular linear differential equations and its effectiveness is demonstrated for $4^{\text {th }}$ order equations with an oscillating potential.
\end{abstract}

Keywords: spectral theory of differential operators, asymptotic formulae for solutions to differential equations.

Mathematics Subject Classification: $34 \mathrm{~K} 08$

\section{INTRODUCTION}

In the work we develop a new approach for studying asymptotic formulae of the fundamental system of solutions (FSS) as $x \rightarrow+\infty$ of linear differential equations with a spectral parameter $\lambda \in \mathbb{C}:$

$$
-\frac{d^{n}}{d x^{n}} y(x, \lambda)+\lambda q(x) y(x, \lambda)=0, \quad x \in\left[x_{0}, \infty\right)
$$

It is well-known that if potential $q(x)$ has a "regular" behavior as $x \rightarrow+\infty$, these equations can be reduced to a system of linear differential equations with almost diagonal matrix. Then, by means of the well-known Levinson theorem [1] one can construct the asymptotics for the solutions as $x \rightarrow \infty$. The classes of so-called "regular" potentials $q(x)$ having a regular behavior as $x \rightarrow+\infty$ consists of the functions satisfying Titchmarsch-Levitan kind conditions [1]: $q(x)$ is twice continuously differentiable, $q^{\prime}(x), q^{\prime \prime}(x)$ are sign-definite on $\left[x_{0}, \infty\right)$ and

$$
q(x) \rightarrow+\infty, \quad q^{\prime}(x)=o\left(q^{\gamma}(x)\right), \quad 0<\gamma<1+\frac{1}{n}, \quad x \rightarrow+\infty .
$$

In this case FSS of these equations have the asymptotics [3]:

$$
y_{k}(x, \lambda) \sim \frac{1}{\left[\mu_{k}(x, \lambda)\right]^{\frac{n-1}{2}}} \exp \int_{x_{0}}^{x} \mu_{k}(t, \lambda) d t,
$$

as $x \rightarrow+\infty$, where $\mu_{k}(x, \lambda)$ are the roots of the equation $\mu^{n}-\lambda q(x)=0, k=1, \ldots, n$.

The aim of the present paper is to enlarge the classes of differential equations with non-regular coefficients so that one can find the asymptotic formulae as $x \rightarrow+\infty$ for their solutions.

\footnotetext{
N.F. Valeev, E.A. Nazirova, Ya.T. Sultanaev, On a neW approach for studying asymptotic BEHAVIOR OF SOLUTIONS TO SINGULAR DIFFERENTIAL EQUATIONS.

The work is supported by RFBR, project no. 15-01-01095 ¡iDirect and inverse problems in spectral theory of differential operatorsi $i$.

(C) Valeev N.F., Nazirova E.A., Sultanaev Ya.T. 2015.

Submitted July 24, 2015.
} 
In order to demonstrate the main idea of constructing the asymptotics of FSS by the proposed approach, we consider the following equation:

$$
-\frac{d^{4}}{d x^{4}} y(x, \lambda)+\lambda^{4}(q(x)+h(x)) y(x, \lambda)=0,
$$

where $q(x)$ is a potential of a regular type, and $h(x)$ is a perturbation of a non-regular type not relatively bounded w.r.t. $q(x)$.

The proposed scheme of constructing the asymptotics of FSS is oriented for the classes of perturbation $h(x)$ which can be called "fast oscillating". Examples of such perturbations are the functions

$$
h(x)=\sum \rho_{k}(x) P_{k}\left(\phi_{k}(x)\right)
$$

where $P_{k}(t)$ is a periodic function, and $\rho_{k}(x), \phi_{k}(x)$ are monotonic functions.

We observe that Sturm-Liouville equation with such potentials were studied by many authors ([2]-[5] and others). As a rule, in order to obtain FSS, in these works there was assumed that $q(x)$ and $h(x)$ satisfy the condition: the function $a(x)=\int_{x}^{\infty} h(t) / \sqrt{q(t)} d t$ is integrable at infinity. Under this condition, in works [6], 7] the asymptotics for FSS were obtained by reducing the original differential equation to an integral one followed by applying the principle of contracting mappings.

\section{MAIN RESULT}

Let us consider the following equation

$$
-\frac{d^{4}}{d x^{4}} y(x, \lambda)+\lambda^{4}(q(x)+h(x)) y(x, \lambda)=0
$$

where $\lambda \in \mathbb{C}, 0 \leqslant \arg \lambda<\pi / 2, x \in[0, \infty)$.

Potential $q(x)$ is twice continuously differentiable function and satisfy regularity conditions of Titchmarsh-Levitan kind, namely, $q^{\prime \prime}(x), q^{\prime}(x)$ are sign-definite for sufficiently large $x$ and

$$
q(x) \rightarrow+\infty, \quad q^{\prime}(x)=o\left(q^{\gamma}(x)\right), \quad 0<\gamma<5 / 4, \quad x \rightarrow+\infty .
$$

The spectral parameter in equation (2.1) is introduced in such form in order to simplify further calculations.

From equation (2.1) to a system of linear first order differential equations by introducing the vector column $Y=\left(y, y^{\prime}, y^{\prime \prime}, y^{\prime \prime \prime}\right)$ :

$$
\begin{gathered}
Y^{\prime}=A \cdot Y=\left(A_{0}+A_{1}\right) Y \\
A_{0}(x, \lambda)=\left(\begin{array}{cccc}
0 & 1 & 0 & 0 \\
0 & 0 & 1 & 0 \\
0 & 0 & 0 & 1 \\
\lambda^{4} q(x) & 0 & 0 & 0
\end{array}\right), A_{1}(x, \lambda)=\left(\begin{array}{cccc}
0 & 0 & 0 & 0 \\
0 & 0 & 0 & 0 \\
0 & 0 & 0 & 0 \\
\lambda^{4} h(x) & 0 & 0 & 0
\end{array}\right) .
\end{gathered}
$$

In order to construct the asymptotics for FSS of equation (2.2), we reduce it to "almost diagonal" form [1].

The eigenvalues of matrix $A_{0}(x, \lambda)$ read as $\mu_{1}(x, \lambda)=\lambda \mu(x), \quad \mu_{2}(x, \lambda)=i \lambda \mu(x), \quad \mu_{3}(x, \lambda)=-\lambda \mu(x), \quad \mu_{4}(x, \lambda)=-i \lambda \mu(x), \quad \mu(x):=q^{1 / 4}(x)$.

Let

$$
T(x, \lambda)=\left(\begin{array}{cccc}
(\lambda \mu)^{-3} & 0 & 0 & 0 \\
0 & (\lambda \mu)^{-2} & 0 & 0 \\
0 & 0 & (\lambda \mu)^{-1} & 0 \\
0 & 0 & 0 & 1
\end{array}\right)\left(\begin{array}{cccc}
1 & i & -1 & -i \\
1 & -1 & 1 & -1 \\
1 & -i & -1 & i \\
1 & 1 & 1 & 1
\end{array}\right) .
$$

Then $T(x, \lambda)$ reduces $A_{0}(x, \lambda)$ to the diagonal form:

$$
T^{-1} A_{0} T=\Lambda(x, \lambda), \quad \Lambda(x, \lambda)=\operatorname{diag}\left\{\mu_{1}(x, \lambda), \mu_{2}(x, \lambda), \mu_{3}(x, \lambda), \mu_{4}(x, \lambda)\right\}=\lambda \mu(x) \Lambda_{0},
$$




$$
\Lambda_{0}=\left(\begin{array}{cccc}
1 & 0 & 0 & 0 \\
0 & i & 0 & 0 \\
0 & 0 & -1 & 0 \\
0 & 0 & 0 & -i
\end{array}\right)
$$

The change

$$
Y=T \cdot Z, \quad Z=\left(z_{1}, z_{2}, z_{3}, z_{4}\right)
$$

transforms system $(2.2)$ to

$$
Z^{\prime}(x, \lambda)=\left(\lambda \mu(x) \Lambda_{0}+\frac{\lambda h(x)}{4 \mu^{3}(x)} G_{0}-\frac{\mu^{\prime}(x)}{2 \mu(x)} F_{0}\right) Z(x, \lambda),
$$

where

$$
\begin{aligned}
& \frac{\lambda h(x)}{4 \mu^{3}(x)} G_{0}=T^{-1} A_{1} T, \quad \frac{\mu^{\prime}(x)}{2 \mu(x)} F_{0}=T^{-1} T^{\prime}, \\
& G_{0}=\left(\begin{array}{cccc}
1 & i & -1 & -i \\
1 & i & -1 & -i \\
1 & i & -1 & -i \\
1 & i & -1 & -i
\end{array}\right), \quad F_{0}=\left(\begin{array}{cccc}
3 & -1+i & -1 & -1-i \\
-1-i & 3 & -1+i & -1 \\
-1 & -1-i & 3 & -1+i \\
-1+i & -1 & -1-i & 3
\end{array}\right)
\end{aligned}
$$

We pass to new variables in system of differential equations (2.4):

$$
\xi=\int_{0}^{x} \mu(t) d t, \quad x=\varphi(\xi), \quad Z(x)=U(\xi)
$$

We obtain the system of equations:

$$
U_{\xi}^{\prime}(\xi, \lambda)=\left(\lambda \Lambda_{0}+\frac{\lambda h(x)}{4 q(x)} G_{0}-\frac{\mu_{x}^{\prime}(x)}{2 \mu^{2}(x)} F_{0}\right) U(\xi, \lambda) .
$$

We denote

On function $\alpha(\xi)$ we impose the condition

$$
\alpha(\xi)=\frac{h(x)}{4 q(x)}, \quad \beta(\xi)=\frac{\mu_{x}^{\prime}(x)}{2 \mu^{2}(x)} .
$$

$$
\left|\int_{\xi}^{\infty} \alpha(s) d s\right|<\infty, \quad \forall \xi>0
$$

and introduce the function

$$
\alpha_{1}(\xi):=\int_{\xi}^{\infty} \alpha(s) d s
$$

We let

By the change we obtain

$$
U(\xi, \lambda)=e^{-\lambda \alpha_{1}(\xi) G_{0}} \cdot V(\xi, \lambda)
$$

$$
V_{\xi}^{\prime}(\xi, \lambda)=\left(\lambda e^{\lambda \alpha_{1}(\xi) G_{0}} \cdot \Lambda_{0} \cdot e^{-\lambda \alpha_{1}(\xi) G_{0}}-\beta(\xi) \cdot e^{\lambda \alpha_{1}(\xi) G_{0}} \cdot F_{0} \cdot e^{-\lambda \alpha_{1}(\xi) G_{0}}\right) V(\xi, \lambda)
$$

Since $G_{0}^{2}=0$, then

$$
V_{\xi}^{\prime}(\xi, \lambda)=\left(\lambda \Lambda_{0}-\lambda^{2} \alpha_{1}(\xi) G_{11}-\beta(\xi) F_{0}+\lambda \beta(\xi) \alpha_{1}(\xi) F_{11}\right) V(\xi, \lambda),
$$

where matrices $G_{11}, F_{11}$ read as

$$
G_{11}=\left(\begin{array}{cccc}
0 & -1-i & 2 & -1+i \\
1-i & 0 & i+1 & -2 \\
2 & i-1 & 0 & -1-i \\
1+i & -2 & -1-i & 0
\end{array}\right), \quad F_{11}=6\left(\begin{array}{cccc}
-1 & -i & 1 & i \\
-1 & -i & 1 & i \\
-1 & -i & 1 & i \\
-1 & -i & 1 & i
\end{array}\right)
$$


Let us assume the following conditions:

$$
\begin{gathered}
\left|\int_{\xi}^{\infty} \alpha_{1}(t) d t\right|<\infty, \quad \xi>0, \\
\alpha_{1}(\xi) \beta(\xi) \in L_{1}(0, \infty) .
\end{gathered}
$$

We rewrite system (2.9) in a simpler form:

$$
V_{\xi}^{\prime}(\xi, \lambda)=\left(\lambda \Lambda_{0}-\lambda^{2} \alpha_{1}(\xi) G_{11}-\beta(\xi) F_{0}+F_{1}(\xi, \lambda)\right) V(\xi, \lambda),
$$

where the entries of the matrix $F_{1}(\xi, \lambda)=\lambda \beta(\xi) \alpha_{1}(\xi) F_{11}$ belong to $L_{1}(0, \infty)$ under conditions (2.7), (2.10), (2.11).

We introduce the function

$$
\alpha_{2}(\xi)=\int_{\xi}^{\infty} \alpha_{1}(s) d s .
$$

We make a change similar to (2.8):

$$
V(\xi, \lambda)=e^{-\lambda^{2} \alpha_{2}(\xi) G_{11}} \cdot R(\xi, \lambda) .
$$

Then for $R(\xi, \lambda)$ we get

$$
R_{\xi}^{\prime}(\xi, \lambda)=e^{\lambda^{2} \alpha_{2}(\xi) G_{11}} \cdot\left(\lambda \Lambda_{0}-\beta(\xi) \cdot F_{0}+F_{1}(\xi, \lambda)\right) \cdot e^{-\lambda^{2} \alpha_{2}(\xi) G_{11}} R(\xi, \lambda) .
$$

Since $G_{11}^{2}=0$, we arrive at the system

$$
R_{\xi}^{\prime}(\xi, \lambda)=\left(\lambda \Lambda_{0}-\beta(\xi) F_{0}+F_{2}(\xi, \lambda)\right) R(\xi, \lambda),
$$

where

$$
F_{2}(\xi, \lambda)=e^{\lambda^{2} \alpha_{2}(\xi) G_{11}} \cdot F_{1}(\xi, \lambda) \cdot e^{-\lambda^{2} \alpha_{2}(\xi) G_{11}}-\lambda^{3} \alpha_{2}(\xi) G_{21}+\frac{\left(\lambda^{3} \alpha_{2}(\xi)\right)^{2}}{2 !} G_{22}+\lambda^{2} \alpha_{2}(\xi) \beta(\xi) F_{21} .
$$

At that, matrices $G_{21}, G_{22}$ read as

$$
G_{21}=\left(\begin{array}{cccc}
0 & -2 & 4 & -2 \\
2 i & 0 & 2 i & -4 i \\
-4 & 2 & 0 & 2 \\
-2 i & 4 i & -2 i & 0
\end{array}\right), \quad G_{22}=\left(\begin{array}{cccc}
8 & 8 i & -8 & -8 i \\
8 & 8 i & -8 & -8 i \\
8 & 8 i & -8 & -8 i \\
8 & 8 i & -8 & -8 i
\end{array}\right)
$$

while matrix $F_{21}$ can be calculated explicitly.

We assume the following condition:

$$
\alpha_{2}(\xi) \in L_{1}(0, \infty)
$$

Then the entries of matrix $F_{2}(\xi, \lambda)$ are integrable over $(0, \infty)$.

We write matrix $\lambda \Lambda_{0}-\beta(\xi) F_{0}$ as

$$
\widetilde{\Lambda}_{0}=\left(\begin{array}{cccc}
\lambda+3 \beta(\xi) & 0 & 0 & 0 \\
0 & i \lambda+3 \beta(\xi) & 0 & 0 \\
0 & 0 & -\lambda+3 \beta(\xi) & 0 \\
0 & 0 & 0 & -i \lambda+3 \beta(\xi)
\end{array}\right), \quad \widetilde{F}_{0}=F_{0}-\left(\begin{array}{cccc}
3 & 0 & 0 & 0 \\
0 & 3 & 0 & 0 \\
0 & 0 & 3 & 0 \\
0 & 0 & 0 & 3
\end{array}\right) .
$$

System (2.14) becomes:

$$
R_{\xi}^{\prime}(\xi, \lambda)=\left(\widetilde{\Lambda}_{0}(\xi, \lambda)-\beta(\xi) \widetilde{F}_{0}+F_{2}(\xi, \lambda)\right) R(\xi, \lambda) .
$$

We make the change

$$
R=\left(I+T_{1}(\xi, \lambda)\right) P, \quad T_{1} \widetilde{\Lambda}_{0}=\widetilde{\Lambda}_{0} T_{1}-\beta(\xi) \widetilde{F}_{0},
$$


that implies

$$
T_{1}(\xi, \lambda)=\frac{\beta(\xi)}{\lambda}\left(\begin{array}{cccc}
0 & -1 & -1 / 2 & -1 \\
i & 0 & i & i / 2 \\
1 / 2 & 1 & 0 & 1 \\
-i & -i / 2 & -i & 0
\end{array}\right)
$$

By transformations we arrive at the system:

$$
P_{\xi}^{\prime}=\widetilde{\Lambda}_{0} P-\left(I+T_{1}\right)^{-1} T_{1}^{\prime} P+\left(I+T_{1}\right)^{-1} F_{2}\left(I+T_{1}\right) P .
$$

We observe that under the regularity of function $q(x)$, in accordance with [1], $\beta^{\prime} \in L_{1}(0, \infty)$. Hence, by conditions (2.7), (2.10), (2.11), (2.15), system (2.17) has $L$-diagonal form and therefore, we can apply Levinson theorem [1] to this system. In accordance with this theorem, the main contribution to the asymptotics of FSS as $x \rightarrow+\infty$ is made by diagonal matrix $\widetilde{\Lambda}_{0}$. Thus, as $x \rightarrow+\infty$, FSS of system of differential equation (2.17) has the following asymptotics:

$$
P(\xi, \lambda)=\exp \left\{\int_{0}^{\xi} \widetilde{\Lambda}_{0}(t, \lambda) d t\right\} \cdot(I+C \cdot o(1)) .
$$

Here matrix $C$ read as:

$$
C=\left(\begin{array}{llll}
1 & 1 & 1 & 1 \\
1 & 1 & 1 & 1 \\
1 & 1 & 1 & 1 \\
1 & 1 & 1 & 1
\end{array}\right)
$$

Returning back to system (2.2) from system (2.17), in view of change of variables (2.3), (2.5), (2.8), (2.12) we obtain the following statement.

Theorem 1. Suppose that in equation (2.1) potential $q(x)$ is twice differentiable function satisfying the conditions: $q^{\prime \prime}(x), q^{\prime}(x)$ are sign-definite for sufficiently large $x$ and

and the functions

$$
q(x) \rightarrow+\infty, \quad \frac{d}{d x} q(x)=o\left(q^{\gamma}(x)\right), \quad 0<\gamma<5 / 4,
$$

$$
\alpha_{1}(\xi)=\int_{\xi}^{\infty} \frac{h(\varphi(s))}{4 q(\varphi(s))} d s, \quad \beta(\xi)=\frac{\frac{d}{d x} q(x)}{32 q^{5 / 4}(x)}, \quad \xi=\int_{0}^{x} q^{1 / 4}(t) d t, \quad x=\varphi(\xi)
$$

satisfy the conditions

$$
\alpha_{1}(\xi) \beta(\xi) \in L_{1}(0,+\infty), \quad \alpha_{2}(\xi)=\int_{\xi}^{\infty} \alpha_{1}(t) d t \in L_{1}(0,+\infty) .
$$

Then equation (2.1) has FSS such that for each fixed $\lambda \neq 0$ as $x \rightarrow+\infty$ the vector $Y(x, \lambda)=$ $\left(y(x, \lambda), y^{\prime}(x, \lambda), y^{\prime \prime}(x, \lambda), y^{\prime \prime \prime}(x, \lambda)\right)$ satisfies the asymptotic formulae:

$$
Y(x, \lambda)=T(x, \lambda) \cdot e^{-\lambda \alpha_{1}(\xi) G_{0}-\lambda^{2} \alpha_{2}(\xi) G_{11}}\left(I+T_{1}(\xi, \lambda)\right) \cdot e^{-\int_{0}^{\xi} \tilde{\Lambda}_{0}(t, \lambda) d t} \cdot(I+C \cdot o(1)) .
$$

\section{EXAMPLE}

Let us provide a typical example of functions $q(x), h(x)$ satisfying all the assumptions of the proven theorem. At that, all the methods known before do not allow one to obtain the asymptotic formulae for the solutions to equation (2.1) with such coefficients since for such methods require the absence of oscillating function $h(x)$. We let

$$
h(x)=(1+x)^{\alpha} \sin (1+x)^{\beta}, \quad q(x)=(1+x)^{\alpha} .
$$


Then the hypothesis of the theorem in the previous section is satisfied if

$$
\beta>\frac{3 \alpha}{8}+\frac{3}{2},
$$

while the asymptotic formulae for FSS of system $(2.2)$ as $x \rightarrow+\infty$ read as

$$
Y(x, \lambda) \sim(1+x)^{-3 \alpha / 8} T(x, \lambda) e^{-\Lambda_{0} \xi-\lambda \alpha_{1}(\xi) G_{0}-\lambda^{2} \alpha_{2}(\xi) G_{11}},
$$

where

$$
\xi=\frac{(1+x)^{\alpha / 4+1}-1}{\alpha / 4+1}
$$

\section{BIBLIOGRAPHY}

1. M.A. Najmark. Linear differential operators. Nauka, Moscow (1969). (in Russian).

2. A.G. Kostyuchenko, I.S. Sargsyan. Distribution of eigenvalues (self-adjoint ordinary differential operators). Nauka, Moscow (1979). (in Russian).

3. M.V. Fedoryuk. Asymptotic analysis: linear ordinary differential equations. Moscow, Nauka (1983). [Springer-Verlag, Berlin (1993).]

4. Kh.Kh. Murtazin, Ya.T. Sultanaev. Formulas for the distribution of eigenvalues of nonsemibounded Sturm-Liouville operators // Matem. Zametki. 28:4, 545-553 (1980). [Math. Notes. 28:4, 733-737 (1980).]

5. N.F. Valeev, Ya.T. Sultanaev On the deficiency indices of a singular Sturm-Liouville operator with a rapidly oscillating perturbation // Doklady Math. 62:2, 271-273 (2000).

6. N.K. Makina, É.A. Nazirova, Ya.T. Sultanaev. On the methods of study of the asymptotic behavior of solutions of singular differential equations // Matem. Zametki. 96:3, 627-632 (2014). [Math. Notes. 96:3, 603-608 (2014).]

7. N.F. Valeev, É.A. Nazirova and Ya.T. Sultanaev. Distribution of the eigenvalues of singular differential operators in a space of vector-functions // Trudy Mosk. Matem. Obsch. 75:2, 107-123 (2014). [Transact. Mosc. Math. Soc. 75:2, 89-102 (2014).]

8. Ya.T. Sultanaev. On asymptotics of spectrum of differential operator in space of vector functions // Differ. Uravn. 10:9, 1673-1683 (1974). (in Russian).

9. R.S. Ismagilov. Asymptotic properties of the spectrum of a differential operator in a space of vectorvalued functions // Matem. Zametki. 9:6, 667-675 (1971). [Math. Notes. 9:6, 387-392 (1971).]

Nurmukhamet Fuatovich Valeev,

Institute of Mathematics CC USC RAS,

Chernyshevskii str. 112,

450008, Ufa, Russia

E-mail: ValeevNF@yandex.ru

Elvira Airatovna Nazirova,

Bashkir State University,

Zaki Validi str. 32,

450076, Ufa, Russia

E-mail: ellkid@gmail.com

Yaudat Talgatovich Sultanaev,

Akhmulla Bashkir State Pedagogical University,

October rev. str. 3a,

450000, Ufa, Russia

E-mail: sultanaevYT@gmail.com 